\title{
COMPUTATION OF CRITICAL PARAMETERS FOR A PROBLEM IN COMBUSTION THEORY
}

\author{
K. K. TAM'
}

(Received 22 May, 1981; revised 5 October, 1981)

\begin{abstract}
An iteration scheme previously obtained by the author is used to study the dependence of criticality on initial data and the parameters in a combustion problem. Numerical results are presented for a slab, a cylinder and a sphere. These are compared with the results of previous workers.
\end{abstract}

\section{Introduction}

A central equation in the study of auto-catalytic reactions is

$$
\frac{\partial \theta}{\partial t}=\nabla^{2} \theta+\delta F(\theta) \text { in } D
$$

subject to

$$
\theta(\mathbf{x}, 0)=h(\mathbf{x}) ; \quad \theta=0 \text { on } \partial D,
$$

where $F(\theta)=\exp (\alpha \theta /(\alpha+\theta)), \theta$ is the temperature, $\mathbf{x}, t$ are the spatial and time variables respectively and $\delta$ and $\alpha$ are positive parameters.

Recently, Tam [3], [4] considered the role of the initial data in a problem in combustion theory, for the special geometries of an infinite slab, an infinite circular cylinder, and a sphere; and the disappearance of criticality was examined using the upper and lower solutions constructed in [5].

In this note, we use the iteration scheme obtained in [3] and [4] to study more extensively the dependence of criticality on the initial data as well as the

\footnotetext{
'McGill University, Department of Mathematics, Burnside Hall-Room 1005, 805 Sherbrooke Street West, Montreal, Quebec H3A 2K6, Canada.

(c) Copyright Australian Mathematical Society 1982
} 
parameters. Since the treatment is numerical, some of the approximations used in [3] and [4] in order to give analytic results are obviated. The improvement thus achieved is especially significant when $\alpha$ is close to 4 . In the next section, we quote the iteration scheme, then we present the numerical results in tables for the case of the slab, the cylinder and the sphere.

\section{Criticality dependence on initial data and parameters}

We have from [3] and [4] the result

$$
\theta_{n+1}(r, t) \sim \delta u_{1}(r) \int_{T}^{t} \exp \left[-\lambda_{1}^{2}(t-s)\right] u_{1}(\xi) \cdot F\left(\theta_{n}(\xi, s)\right) d s
$$

where $r$ denotes the only spatial variable, $\lambda_{1}^{2}$ and $u_{1}(r)$ the first eigenvalue and normalized eigenfunction of the eigenvalue problem

$$
\begin{aligned}
& \nabla^{2} u_{k}+\lambda_{k}^{2} u_{k}=0 \\
& u_{k}=0 \quad \text { on } \partial D
\end{aligned}
$$

and

$$
u_{1}(\xi) \cdot F\left(\theta_{n}(\xi, s)\right)=\int_{D} u_{1}(\xi) F\left(\theta_{n+1}(\xi, s)\right) d \xi
$$

with $D$ denoting the region under consideration. If, for $t>T$, we have $u_{1}(\xi)$. $F\left(\theta_{n}(\xi, s)\right) \sim K_{n}$ for some $n$, where $K_{n}$ is independent of $s$, then for $t \gg T$ we have

$$
\theta_{n+1}(r, t) \sim \frac{K_{n} \delta}{\lambda_{1}^{2}} u_{1}(r)
$$

Using the above, we consider, for $s$ large,

$$
u_{1}(\xi) \cdot F\left(\theta_{n}(\xi, s)\right) \sim K_{n+1},
$$

and from which we have, for $t$ large,

$$
\theta_{n+2}(r, t) \sim \frac{K_{n+1} \delta}{\lambda_{1}^{2}} u_{1}(r)
$$

Thus, the steady state reached is deduced by comparing $K_{n+1}$ with $K_{n}$. In [3] and [4] we made approximations in the evaluation of $K_{n+1}$. In this note, we obtain $K_{n+1}$ numerically, from which we determine all other critical parameters. Criticality disappears when $\alpha<\tilde{\alpha}$, which depends on geometry, but in all cases is slightly larger than 4. When $\alpha>\tilde{\alpha}$, there is a unique steady state when $\delta<\delta_{e}(\alpha)$ or when $\delta>\delta_{c r}(\alpha)$. For $\delta_{e}<\delta<\delta_{c r}$, there are two stable steady states, the one with the larger maximum referred to as super-critical, and the one with the smaller maximum referred to as sub-critical. In this range, we determine $v^{*}(\delta, \alpha)$ such that for $K_{n}>\delta^{-1} v^{*}$, the super-critical state is reached while for $K_{n}<\delta^{-1} v^{*}$, the sub-critical state is reached. The limiting values of $K_{n}$, namely $\bar{K}_{\infty}$ and $\tilde{K}_{\infty}$ are also determined. These quantities are presented in tables. 
TABLE 1

Values of $\tilde{K}_{\infty}$, and $\bar{K}_{\infty}$ for some values of $\alpha$ and $\delta$ for the slab.

\begin{tabular}{|c|c|c|c|c|}
\hline$\alpha$ & $\delta$ & $\tilde{K}_{\infty}$ & $v^{*}$ & $\bar{K}_{\infty}$ \\
\hline 100 & $\begin{aligned} \delta_{e}= & 1.02 E-38 \\
& 0.1 \\
& 1.0 \\
& 2.0 \\
\delta_{c r}= & 3.546\end{aligned}$ & $\begin{array}{l}.920 E-38 \\
0.091 \\
1.01 \\
2.35 \\
8.51\end{array}$ & $\begin{array}{l}83260 \\
54.0 \\
29.5 \\
20.9 \\
8.51\end{array}$ & $\begin{array}{l}83260 \\
0.243 E 43 \\
0.242 E 44 \\
0.484 E 44 \\
0.858 E 44\end{array}$ \\
\hline 70 & $\begin{aligned} \delta_{e}= & 5.33 E-26 \\
& 0.1 \\
& 1.0 \\
& 2.0 \\
\delta_{c r}= & 3.5633\end{aligned}$ & $\begin{array}{l}0.48 E-25 \\
0.091 \\
1.01 \\
2.35 \\
8.61\end{array}$ & $\begin{array}{l}40429 \\
56.2 \\
30.3 \\
21.3 \\
8.61\end{array}$ & $\begin{array}{l}40429 \\
0.2265 E 30 \\
0.226 E 31 \\
0.4529 E 31 \\
0.806 E 31\end{array}$ \\
\hline 40 & $\begin{aligned} \delta_{e}= & 1.837 E-13 \\
& 0.1 \\
& 1.0 \\
& 2.0 \\
\delta_{c r}= & 3.6\end{aligned}$ & $\begin{array}{l}0.1656 E-12 \\
0.09 \\
1.01 \\
2.35 \\
8.81\end{array}$ & $\begin{array}{l}12892 \\
62.2 \\
32.2 \\
22.4 \\
8.81\end{array}$ & $\begin{array}{l}12892 \\
0.212 E 17 \\
0.2119 E 18 \\
0.4238 E 18 \\
0.764 E 18\end{array}$ \\
\hline 20 & $\begin{aligned} \delta_{e}= & 2.164 E-5 \\
& 0.1 \\
& 1.0 \\
& 2.0 \\
\delta_{c r}= & 3.708\end{aligned}$ & $\begin{array}{l}0.1948 E-4 \\
0.91 \\
1.01 \\
2.34 \\
9.34\end{array}$ & $\begin{array}{l}3039 \\
84.0 \\
37.9 \\
25.5 \\
9.34\end{array}$ & $\begin{array}{l}3039 \\
0.4367 E 8 \\
0.4368 E 9 \\
0.8790 E 9 \\
0.162 E 10\end{array}$ \\
\hline 15 & $\begin{aligned} \delta_{e}= & 1.77 E-3 \\
& 0.5 \\
& 1.6 \\
& 2.5 \\
\delta_{c r}= & 3.77\end{aligned}$ & $\begin{array}{l}0.1593 E-2 \\
0.475 \\
1.75 \\
3.22 \\
9.74\end{array}$ & $\begin{array}{l}1639 \\
59.6 \\
33.1 \\
23.3 \\
9.74\end{array}$ & $\begin{array}{l}1639 \\
0.1469 E 7 \\
0.4706 E 7 \\
0.7355 E 7 \\
0.11 E 8\end{array}$ \\
\hline 8 & $\begin{aligned} \delta_{e}= & 0.51 \\
& 1.0 \\
& 2.0 \\
& 3.0 \\
\delta_{c r}= & 4.06\end{aligned}$ & $\begin{array}{l}0.4846 \\
1.01 \\
2.33 \\
4.25 \\
11.6\end{array}$ & $\begin{array}{l}390 \\
96.6 \\
46.8 \\
28.5 \\
11.6\end{array}$ & $\begin{array}{l}390 \\
1970 \\
4678 \\
7366 \\
10220\end{array}$ \\
\hline 4.5 & $\begin{aligned} \delta_{e}= & 4.457 \\
& 4.7 \\
\delta_{c r}= & 4.88\end{aligned}$ & $\begin{array}{l}9.875 \\
12.52 \\
19.8\end{array}$ & $\begin{array}{l}73 \\
35.15 \\
19.8 \\
\end{array}$ & $\begin{array}{l}73 \\
126 \\
148\end{array}$ \\
\hline$\tilde{\alpha}=4.069$ & $\delta_{e}=5.227=\delta_{c r}$ & 34 & 34 & 34 \\
\hline
\end{tabular}


TABLE 2

Values of $\tilde{K}_{\infty}, v^{*}$, and $\bar{K}_{\infty}$ for some values of $\alpha$ and $\delta$ for a sphere.

\begin{tabular}{|c|c|c|c|c|}
\hline$\alpha$ & $\delta$ & $\tilde{K}_{\infty}$ & $v^{*}$ & $\bar{K}_{\infty}$ \\
\hline 100 & $\begin{aligned} \delta_{e}= & 1.08 E-38 \\
& 0.1 \\
& 1.0 \\
& 2.0 \\
\delta_{c r}= & 3.33\end{aligned}$ & $\begin{array}{l}0.1685 E-37 \\
0.161 \\
1.79 \\
4.197 \\
13.5\end{array}$ & $\begin{array}{l}138080 \\
74.19 \\
42.13 \\
30.01 \\
13.5\end{array}$ & $\begin{array}{l}138080 \\
0.429 E 43 \\
0.4290 E 44 \\
0.8579 E 44 \\
0.1430 E 45\end{array}$ \\
\hline 70 & $\begin{aligned} & \delta_{e}= 5.63 E-26 \\
& 0.1 \\
& 1.0 \\
& 2.0 \\
& \delta_{c r}= 3.352 \\
&\end{aligned}$ & $\begin{array}{l}0.899 E-25 \\
0.16 \\
1.79 \\
4.195 \\
13.6\end{array}$ & $\begin{array}{l}66989 \\
77.3 \\
43.2 \\
30.74 \\
13.6\end{array}$ & $\begin{array}{l}66989 \\
0.4001 E 30 \\
0.4014 E 31 \\
0.8025 E 31 \\
0.1345 E 32\end{array}$ \\
\hline 40 & $\begin{aligned} \delta_{e}= & 1.94 E-13 \\
& 0.1 \\
& 1.0 \\
& 2.0 \\
\delta_{c r}= & 3.395\end{aligned}$ & $\begin{array}{l}0.3099 E-12 \\
0.16 \\
1.79 \\
4.19 \\
14.0\end{array}$ & $\begin{array}{l}21326 \\
86.4 \\
46.2 \\
32.4 \\
14.0\end{array}$ & $\begin{array}{l}21326 \\
0.3756 E 17 \\
0.3760 E 18 \\
0.7512 E 18 \\
0.1275 E 19\end{array}$ \\
\hline 20 & $\begin{aligned} \delta_{e}= & 2.275 E-5 \\
& 0.1 \\
& 1.0 \\
& 2.0 \\
\delta_{c r}= & 3.5033\end{aligned}$ & $\begin{array}{l}0.363 E-4 \\
0.16 \\
1.79 \\
4.18 \\
14.9\end{array}$ & $\begin{array}{l}5005 \\
119.6 \\
55.1 \\
37.3 \\
14.9\end{array}$ & $\begin{array}{l}5005 \\
0.7750 E 8 \\
0.7708 E 9 \\
0.1549 E 10 \\
0.271 E 10\end{array}$ \\
\hline 15 & $\begin{aligned} \delta_{e}= & 1.856 E-3 \\
& 0.1 \\
& 1.0 \\
& 2.0 \\
\delta_{c r}= & 3.5813\end{aligned}$ & $\begin{array}{l}0.296 E-2 \\
0.16 \\
1.79 \\
4.16 \\
15.6\end{array}$ & $\begin{array}{l}2690 \\
162.2 \\
63.4 \\
41.5 \\
15.6\end{array}$ & $\begin{array}{l}2690 \\
0.5160 E 6 \\
0.5210 E 7 \\
0.1043 E 8 \\
0.187 E 8\end{array}$ \\
\hline 8 & $\begin{aligned} \delta_{e}= & 0.5296 \\
& 1.2 \\
& 2.2 \\
\delta_{c r}= & 3.902\end{aligned}$ & $\begin{array}{l}0.895 \\
2.2 \\
4.7 \\
19\end{array}$ & $\begin{array}{l}632 \\
123.8 \\
64.2 \\
19\end{array}$ & $\begin{array}{l}632 \\
0.421 E 4 \\
0.896 E 4 \\
0.1703 E 5\end{array}$ \\
\hline 4.5 & $\begin{aligned} \delta_{e}= & 4.509 \\
& 4.62 \\
\delta_{c r}= & 4.767\end{aligned}$ & $\begin{array}{l}19.8 \\
22.63 \\
35.2\end{array}$ & $\begin{array}{l}107 \\
64.04 \\
35.2\end{array}$ & $\begin{array}{l}107 \\
162.9 \\
1986\end{array}$ \\
\hline$\tilde{\alpha}=4.192$ & $\delta_{e}=5.034=\delta_{c r}$ & 57 & 57 & 57 \\
\hline
\end{tabular}


TABLE 3

Values of $\tilde{K}_{\infty}, v^{*}$ and $\bar{K}_{\infty}$ for some values of $\alpha$ and $\delta$ for the cylinder.

\begin{tabular}{|c|c|c|c|c|}
\hline$\alpha$ & $\delta$ & $\tilde{K}_{\infty}$ & $v^{*}$ & $\bar{K}_{\infty}$ \\
\hline 100 & $\begin{aligned} \delta_{e}= & 8.376 E-39 \\
& 0.1 \\
& 1.0 \\
\delta_{c r}= & 2.01\end{aligned}$ & $\begin{array}{l}0.124 E-37 \\
0.1503 \\
1.838 \\
7.71\end{array}$ & $\begin{array}{l}105791 \\
41.06 \\
19.80 \\
7.71\end{array}$ & $\begin{array}{l}105791 \\
0.3148 E 43 \\
0.3148 E 44 \\
0.633 E 44\end{array}$ \\
\hline 70 & $\begin{aligned} \delta_{e}= & 4.365 E-26 \\
& 0.1 \\
& 1.0 \\
\delta_{c r}= & 2.0194\end{aligned}$ & $\begin{array}{l}0.6445 E-25 \\
0.1503 \\
1.837 \\
7.79\end{array}$ & $\begin{array}{l}51450 \\
42.56 \\
20.25 \\
7.79\end{array}$ & $\begin{array}{l}51450 \\
0.2946 E 30 \\
0.2946 E 31 \\
0.595 E 31\end{array}$ \\
\hline 40 & $\begin{aligned} \delta_{e}= & 1.502 E-13 \\
& 0.1 \\
& 1.0 \\
\delta_{c r}= & 2.045\end{aligned}$ & $\begin{array}{l}0.2217 E-12 \\
0.1503 \\
1.836 \\
7.96\end{array}$ & $\begin{array}{l}16457 \\
46.95 \\
21.42 \\
7.96\end{array}$ & $\begin{array}{l}16457 \\
0.2759 E 17 \\
0.2759 E 18 \\
0.564 E 18\end{array}$ \\
\hline 20 & $\begin{aligned} \delta_{e}= & 1.721 E-5 \\
& 0.1 \\
& 1.0 \\
\delta_{c r}= & 2.1092\end{aligned}$ & $\begin{array}{l}0.254 E-4 \\
0.1503 \\
1.833 \\
8.57\end{array}$ & $\begin{array}{l}3826 \\
62.23 \\
24.8 \\
8.57\end{array}$ & $\begin{array}{l}3826 \\
0.5767 E 8 \\
0.5767 E 9 \\
0.1216 E 10\end{array}$ \\
\hline 15 & $\begin{aligned} \delta_{e}= & 1.367 E-3 \\
& 0.1 \\
& 1.0 \\
\delta_{c r}= & 2.1552\end{aligned}$ & $\begin{array}{l}0.202 E-2 \\
0.1503 \\
1.83 \\
8.9\end{array}$ & $\begin{array}{l}2021 \\
80.45 \\
27.8 \\
8.9\end{array}$ & $\begin{array}{l}2021 \\
0.3920 E 6 \\
0.3944 E 7 \\
0.8506 E 7\end{array}$ \\
\hline 8 & $\begin{aligned} \delta_{e}= & 0.3578 \\
& 0.8 \\
& 1.6 \\
\delta_{c r}= & 2.3456\end{aligned}$ & $\begin{array}{l}0.564 \\
1.39 \\
3.53 \\
10.6\end{array}$ & $\begin{array}{l}448 \\
65.8 \\
29.5 \\
10.6\end{array}$ & $\begin{array}{l}448 \\
0.2260 E 4 \\
0.5290 E 4 \\
0.811 E 4\end{array}$ \\
\hline 4.5 & $\begin{aligned} \delta_{e} & =2.8077 \\
\delta_{c r} & =2.8625\end{aligned}$ & $\begin{array}{l}14 \\
17.9\end{array}$ & $\begin{array}{l}77.5 \\
17.9\end{array}$ & $\begin{array}{l}77.5 \\
91\end{array}$ \\
\hline$\tilde{\alpha}=4.002$ & $\delta_{e}=3.205=\delta_{c r}$ & 33 & 33 & 33 \\
\hline
\end{tabular}

\section{Results for the slab, the sphere and the cylinder}

For the slab, we have $0 \leqslant r \leqslant 1, \lambda_{1}=\pi$ and $u_{1}(r)=\sqrt{2} \sin \pi r$. Results are presented in Table 1. We start with $\alpha=100$, giving the values of $\delta_{e}$ and $\delta_{c r}$. At 
$\delta=\delta_{e}, v^{*}$ coincides with $\bar{K}_{\infty}$ and at $\delta=\delta_{c r}, \tilde{K}_{\infty}$ coincides with $v^{*}$. For $\delta_{e}<\delta<$ $\delta_{c r}, v^{*}$ is the threshold parameter. We decrease the value of $\alpha$ until we reach $\tilde{\alpha}$ at which $\delta_{e}$ and $\delta_{c r}$ coincide.

For the sphere, we have $0 \leqslant r \leqslant 1, \lambda_{1}=\pi$, and $u_{1}(r)=\sin \pi r /(r \sqrt{2 \pi})$. Results are presented in Table 2.

For the cylinder, we have $0 \leqslant r \leqslant 1, \lambda_{1}=2.405$ and $u_{1}(r)=J_{0}\left(\lambda_{1} r\right) / J_{1}\left(\lambda_{1}\right) \sqrt{\pi}$. Results are presented in Table 3.

\section{Concluding remarks}

Using a method proposed by Kordylewski [2], Fenaughty et al. [1] recently obtained numerical critical values for the parameters $\delta$ and $\alpha$ for the three simple geometries. Their treatment is also numerical. Table 4 shows a comparison of the present result with theirs.

TABLE 4

Critical parameters as determined by the two methods

\begin{tabular}{l|c|c} 
& Present result & Fenaughty et al. \\
\hline $\begin{array}{l}\text { Infinite slab } \\
\text { (after adjusting for }\end{array}$ & $\delta_{c r}=5.227$ & $\delta_{c r}=5.2294$ \\
different scaling) & $\tilde{\alpha}=4.069$ & $\tilde{\alpha}=4.0687$ \\
\hline Infinite cylinder & $\delta_{c r}=3.205$ & $\delta_{c r}=3.00617$ \\
& $\tilde{\alpha}=4.002$ & $\dot{\alpha}=4.1304$ \\
\hline Sphere & $\delta_{c r}=5.034$ & $\delta_{c r}=5.04081$ \\
& $\tilde{\alpha}=4.192$ & $\tilde{\alpha}=4.1876$ \\
\hline
\end{tabular}

Despite the slight discrepancy, it must be emphasized that the present integral equation approach produces not just $\tilde{\alpha}$ and $\delta_{c r}$, but $\delta_{e}$ as well. For any given $\alpha>\tilde{\alpha}$, and $\delta_{e}<\delta<\delta_{c r}$, it also produces the number $v^{*}$, which is used to determine whether an arbitrarily given initial $\theta(r, 0)$ leads to the super-critical or sub-critical steady state.

Another observation that is perhaps worth making is that if one starts with the elliptic equation obtained from the governing equation by dropping the $\partial \theta / \partial t$ term and think of its multiple solutions as possible steady states, then since there may be more than two "steady state" solutions, it is difficult to attach the label 
"super-critical" or "sub-critical" to them. There is no such difficulty if one starts with the parabolic equation. Since the initial value problem has a unique solution, the steady state is either super-critical or sub-critical, depending on the initial data $\theta(r, 0)$. The present work does in fact provide estimates on these two steady states, and the criterion on $\theta(r, 0)$ that determines which one is reached.

\section{Acknowledgements}

The author is grateful to Dr. G. Wake for his remarks pointing out an error in [5] cited in the References. A forthcoming letter to the Zeitschrift für Angewandte Mathematik und Physik by Dr. Wake contains relevant material. Continuing support from the Natural Science and Engineering Research Council of Canada under Grant A-5228 is acknowledged.

\section{References}

[1] K. F. Fenaughty, A. A. Lacey and C. G. Wake, "The disappearance of criticality for small activation energy with arbitrary Biot number", Combustion and Flame (to appear).

[2] W. Kordylewski, "Degenerate critical points of thermal explosion", Komunikat $I-20 / K-020 / 78$, Technical University of Wroclaw, (1978).

[3] K. K. Tam, "Initial data and criticality for a problem in combustion theory", J. Math. Anal. Applic. 77 (1980), 626-634.

[4] K. K. Tam, "On the influence of the initial data in a combustion problem", J. Austral. Math. Soc. B22 (1980), 193-209.

[5] K. K. Tam, "On the disappearance of criticality in the theory of thermal ignition", $Z$. Angew. Math. Phys. 31 (1980), 762-766. 